\title{
Reidemeister torsion of Seifert fibered homology lens spaces and Dehn surgery
}

\author{
TERUHISA KADOKAMI
}

\begin{abstract}
We provide necessary conditions on the Alexander polynomial of a knot $K$ in a homology sphere and on surgery coefficients $p / q$ for the surgered manifold to be a Seifert fibered space over $S^{2}$. As an application, we show that no $p / q$-surgery with $p>3$ on a knot in a homology sphere with the same Alexander polynomial as the figure eight knot can produce a Seifert fibered space with base $S^{2}$. The main tool is the abelian Reidemeister torsion.
\end{abstract}

55R55, 19J10, 57Q10, 57M25

\section{Introduction}

The Reidemeister torsion of manifolds has been studied since 1930's (see Turaev [19]). In particular, the Reidemeister torsion of Seifert fibered rational homology sphere is studied in Némethi and Nicolaescu [12]. In this paper, we study the Reidemeister torsion of Seifert fibered homology lens space over $S^{2}$. It is well-known that every Seifert fibered homology lens space has $S^{2}$ or $\mathbb{R} P^{2}$ as its base space, and every Seifert fibered space over $S^{2}$ has a framed link presentation as in Figure 1, where $p_{i} \geq 2$ for each $i$. We call $p_{i}$ the multiplicity of the singular fiber. See Orlik [14] or Saveliev [16], for example. Thus the object of our study is such a 3-manifold that has the framed link presentation of Figure 1 and has a finite cyclic first homology group. We extract information on multiplicities of singular fibers from Reidemeister torsion by using the norm and order of homology lens spaces which we introduced in [6], and apply the information to the Seifert surgery problem to determine when Dehn surgery yields a Seifert fibered space.

Throughout this paper, $\zeta_{d}$ denotes a primitive $d$-th root of unity, $H_{1}(X)$ denotes the first homology group of $X$ with integer coefficients, and the operation in $H_{1}(X)$ is written multiplicatively unless otherwise stated. We also use the following notation:

- Let $A$ and $B$ be elements of $\mathbb{Q}\left(\zeta_{d}\right)$. Then $A \doteq B$ means an equality of $A$ and $B$ up to multiplication of $\pm \zeta_{d}^{m}(m \in \mathbb{Z})$. 


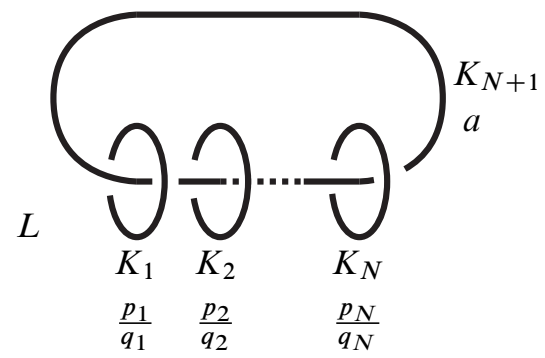

Figure 1: framed link presentation of a Seifert fibered space over $S^{2}$

- Let $\alpha$ be an element of $\mathbb{Q}\left(\zeta_{d}\right)$. Then $N_{d}(\alpha)$ denotes the norm of $\alpha$ associated to the algebraic extension $\mathbb{Q}\left(\zeta_{d}\right)$ over $\mathbb{Q}$.

- Let $x$ be an element of $(\mathbb{Z} / p \mathbb{Z})^{\times}$, the multiplicative group of the ring $\mathbb{Z} / p \mathbb{Z}$. Then $\bar{x}$ denotes the inverse element of $x$.

Let $\Sigma$ be a homology sphere, and $K$ a knot in $\Sigma$. Then $\Delta_{K}(t)$ is the Alexander polynomial of $K$, and $\Sigma(K ; p / q)$ is the result of $p / q$-surgery along $K$. More generally, let $L=K_{1} \cup \cdots \cup K_{n}$ be an $n$-component link in $\Sigma$. Then $\Delta_{L}\left(t_{1}, \cdots, t_{n}\right)$ is the ( $n$-variable) Alexander polynomial of $L$, and $\Sigma\left(L ; p_{1} / q_{1}, \cdots, p_{n} / q_{n}\right)$ is the result of $p_{i} / q_{i}$-surgeries along $K_{i}$ for all $i=1, \cdots, n$. We give a more precise description of $\Sigma\left(L ; p_{1} / q_{1}, \cdots, p_{n} / q_{n}\right)$ in Section 2 .

Let $M$ be a homology lens space with $H_{1}(M) \cong \mathbb{Z} / p \mathbb{Z}(p \geq 2)$, and $T$ a generator of $H_{1}(M)$. Let $d \geq 2$ be a divisor of $p$, and $\psi_{d}: \mathbb{Z}\left[H_{1}(M)\right] \rightarrow \mathbb{Q}\left(\zeta_{d}\right)$ a ring homomorphism such that $\psi_{d}(T)=\zeta_{d}$. Then $\tau^{\psi_{d}}(M) \in \mathbb{Q}\left(\zeta_{d}\right)$, the Reidemeister torsion of $M$ associated to $\psi_{d}$, is determined up to multiplication by $\pm \zeta_{d}^{m}(m \in \mathbb{Z})$ (see Kadokami [7], Nicolaescu [13] or Turaev [18; 19] for details on Reidemeister torsion).

In [6], we introduced the norm and the order of polynomials and homology lens spaces: Let $f(t)$ be a polynomial over $\mathbb{Z}$. We define the $d$-norm of $f(t)$, denoted by $|f(t)|_{d}$, by

$$
|f(t)|_{d}=\left|N_{d}\left(f\left(\zeta_{d}\right)\right)\right|=\left|\prod_{i \in(\mathbb{Z} / d \mathbb{Z})^{\times}} f\left(\zeta_{d}^{i}\right)\right|,
$$

where $d$ is a positive integer. We note that $|f(t)|_{d}$ is a nonnegative integer. For an arbitrary homology lens space $M$, there exists a knot $K$ in a homology sphere $\Sigma$ such that $M=\Sigma(K ; p / q)(p \geq 2)$ (see Boyer and Line [1, Lemma 2.1]). We define the $d$-norm and the $d$-order of $M$, denoted by $|M|_{d}$ and $\|M\|_{d}$ respectively, by

$$
|M|_{d}=\left|\Delta_{K}(t)\right|_{d} \quad \text { and } \quad\|M\|_{d}=\prod_{d^{\prime} \mid d}|M|_{d^{\prime}},
$$


where $d$ is a divisor of $p$. As proved in [6], both $|M|_{d}$ and $\|M\|_{d}$ are invariants of a homology lens space $M$. We note that the norms and orders of a lens space are all 1 because a lens space is the result of surgery along the unknot.

We fix the following setting.

Setting (i) $L=K_{1} \cup \cdots \cup K_{N+1}$ is the $(N+1)$-component link in $S^{3}$ of Figure 1 .

(ii) $M=S^{3}\left(L ; p_{1} / q_{1}, \cdots, p_{N} / q_{N}, a\right)$ is a homology lens space with $H_{1}(M) \cong$ $\mathbb{Z} / p \mathbb{Z}$ where $p \geq 2, p_{i} \geq 2(i=1, \cdots, N), \operatorname{gcd}\left(p_{i}, q_{i}\right)=1$ and $a$ is an integer.

Theorem 1.1 Let $M=S^{3}\left(L ; p_{1} / q_{1}, \cdots, p_{N} / q_{N}, a\right)$ be a Seifert fibered homology lens space as in the setting above. Let $d \geq 2$ be any divisor of $p$ satisfying $\operatorname{gcd}\left(d, p_{i}\right)=$ 1 for all $i=1, \cdots, N$. Then we have the following:

(1) Let $T$ be a generator of $H_{1}(M)$ and $\psi_{d}: \mathbb{Z}\left[H_{1}(M)\right] \rightarrow \mathbb{Q}\left(\zeta_{d}\right)$ a ring homomorphism such that $\psi_{d}(T)=\zeta_{d}$. Let $u$ be an integer such that $\left[m_{N+1}\right]=T^{u}$ in $H_{1}(M)$, where $m_{N+1}$ is a meridian of $K_{N+1}$. Then we have $\operatorname{gcd}(d, u)=1$ and

$$
\tau^{\psi_{d}}(M) \doteq\left(\zeta_{d}^{u}-1\right)^{N-2} \prod_{i=1}^{N}\left(\zeta_{d}^{u \bar{p}_{i}}-1\right)^{-1},
$$

where $\bar{p}_{i}$ is the inverse element of $p_{i}$ in $(\mathbb{Z} / d \mathbb{Z})^{\times}$.

(2) $|M|_{d}=1$.

(3) $\|M\|_{d}=1$.

Theorem 1.2 Let $M=S^{3}\left(L ; p_{1} / q_{1}, \cdots, p_{N} / q_{N}, a\right)$ be a Seifert fibered homology lens space as in the setting above, and assume $N \geq 3$. Let $d \geq 2$ be a common divisor of $p_{1}$ and $p_{2}$. Then we have the following:

(1) $d$ is a divisor of $p, \operatorname{gcd}\left(d, p_{i}\right)=1(i=3, \cdots, N)$, and $\operatorname{gcd}\left(d, q_{i}\right)=1(i=1,2)$.

(2) Let $T$ be a generator of $H_{1}(M)$ and $\psi_{d}: \mathbb{Z}\left[H_{1}(M)\right] \rightarrow \mathbb{Q}\left(\zeta_{d}\right)$ a ring homomorphism such that $\psi_{d}(T)=\zeta_{d}$. Let $u_{1}$ be an integer such that $\left[m_{1}\right]=T^{u_{1}}$ in $H_{1}(M)$, where $m_{1}$ is a meridian of $K_{1}$. Then we have $\operatorname{gcd}\left(d, u_{1}\right)=1$ and

$$
\tau^{\psi_{d}}(M) \doteq p_{3} \cdots p_{N}\left(\zeta_{d}^{u_{1} \bar{q}_{1}}-1\right)^{-1}\left(\zeta_{d}^{u_{1} \bar{q}_{2}}-1\right)^{-1},
$$

where $\bar{q}_{i}(i=1,2)$ is the inverse element of $q_{i}$ in $(\mathbb{Z} / d \mathbb{Z})^{\times}$.

(3) $|M|_{d}=\left(p_{3} \cdots p_{N}\right)^{\varphi(d)}$, where $\varphi(\cdot)$ is the Euler function. 
If $N \leq 2$, then $M$ is a lens space, and hence $\|M\|_{p}=1$.

The next theorem gives a relation between multiplicities of singular fibers and the order of a Seifert fibered homology lens space.

Theorem 1.3 Let $M=S^{3}\left(L ; p_{1} / q_{1}, \cdots, p_{N} / q_{N}, a\right)$ be a Seifert fibered homology lens space as in the setting above. We assume $N \geq 3$. Then we have the following:

(1) $\|M\|_{p}=1$ holds if and only if $\operatorname{gcd}\left(p_{i}, p_{j}\right)=1$ for any pair $\{i, j\}(1 \leq i<j \leq N)$. Moreover it is equivalent to $\operatorname{gcd}\left(p, p_{i}\right)=1$ for all $i=1, \cdots, N$.

(2) $\|M\|_{p} \neq 0,1$ holds if and only if there uniquely exists a pair $\{i, j\}(1 \leq i<j \leq N)$ such that $\operatorname{gcd}\left(p_{i}, p_{j}\right) \geq 2$.

(3) $\|M\|_{p}=0$ holds if and only if there exist at least two distinct pairs $\{i, j\}(1 \leq i<$ $j \leq N)$ and $\{k, h\}(1 \leq k<h \leq N)$ such that $\operatorname{gcd}\left(p_{i}, p_{j}\right) \geq 2$ and $\operatorname{gcd}\left(p_{k}, p_{h}\right) \geq 2$.

As an application of the Reidemeister torsion to Seifert surgery problem, we have the following:

Theorem 1.4 Let $K$ be a knot in a homology sphere $\Sigma$ such that $\Delta_{K}(t)=t^{2}-3 t+1$. The only surgeries on $K$ that may produce a Seifert fibered space with base $S^{2}$ and with $H_{1} \neq\{0\}, \mathbb{Z}$, have coefficients $2 / q$ and $3 / q$, and produce Seifert fibered spaces with three singular fibers. Moreover if the coefficient is $2 / q$, then the set of multiplicities is $\{\alpha, \beta, 5\}$ where $\operatorname{gcd}(\alpha, \beta)=2$, and if the coefficient is $3 / q$, then the set of multiplicities is $\{\alpha, \beta, 4\}$ where $\operatorname{gcd}(\alpha, \beta)=3$.

We note that the Alexander polynomial of the figure eight knot is $t^{2}-3 t+1$. Hence we may consider Theorem 1.4 as an algebraic analogue of the fact shown by W P Thurston that all coefficients of Seifert surgery along the figure-eight knot are $\pm 1, \pm 2$ and \pm 3 [17, Chapter 4]. We mention that the Reidemeister torsion cannot capture the case $p=1$ because Reidemeister torsion of a homology sphere is zero.

In Section 2, we state surgery formulae for the Reidemeister torsion. In Section 3, we prove a key lemma (Lemma 3.4 (1)) derived from the cyclicity of the first homology group. In Section 4 and Section 5, we prove the theorems by using the surgery formula and lemmas in Section 3.

For references on Dehn surgery including Seifert surgery, see Brittenham and Wu [2], Culler, Gordon, Luecke and Shalen [3], Fintushel and Stern [5], Kadokami [6; 7], Kadokami and Yamada [8; 9], Miyazaki and Motegi [10], Moser [11], Orlik [14], Saveliev [16] and Thurston [17]. For Reidemeister torsion, see Kadokami [6; 7], Kadokami and Yamada [8; 9], Nicolaescu [13], de Rham [4], Sakai [15] and Turaev $[18 ; 19]$. 


\section{Surgery formula for Reidemeister torsion}

Lemma 2.5 below is used repeatedly in the later sections. It consists of special cases of a surgery formula for the Reidemeister torsion due to V Turaev, and follows from Lemma 2.1, Lemma 2.2, Lemma 2.3 and Lemma 2.4. We do not give any proofs in this section. For details see Turaev [18; 19], and also Sakai [15] for Lemma 2.6.

Let $R$ be a commutative ring with nonzero identity element. Then we denote the classical ring of quotients by $Q(R)$. Let $X$ be a finite $\mathrm{CW}$-complex. Then the maximal abelian torsion of $X$ denoted by $\tau(X)$ is an element of $Q\left(\mathbb{Z}\left[H_{1}(X)\right]\right)$, which is defined from a chain complex $\mathbf{C}_{*}$ induced by the maximal abelian covering of $X$. Let $\psi: \mathbb{Z}\left[H_{1}(X)\right] \rightarrow R$ be a ring homomorphism. Then a chain complex is induced from $\mathbf{C}_{*}$ and $\psi$. We denote it by $\mathbf{C}_{*}^{\psi}$. The Reidemeister torsion associated to $\psi, \tau^{\psi}(X)$, is defined from $\mathbf{C}_{*}^{\psi}$. It is an element of $Q(R)$ that is determined up to multiplication by an element in $\pm \psi\left(H_{1}(X)\right)$. If $\mathbf{C}_{*}^{\psi}$ is not acyclic, then we define $\tau^{\psi}(X)=0$.

Lemma 2.1 Let $X_{1}$ and $X_{2}$ be subcomplexes of a finite $C W$-complex $X$ such that $X=X_{1} \cup X_{2}$, and $Y=X_{1} \cap X_{2}$. Let $\psi: \mathbb{Z}\left[H_{1}(X)\right] \rightarrow R$ be a ring homomorphism, and let $\psi_{i}: \mathbb{Z}\left[H_{1}\left(X_{i}\right)\right] \rightarrow R(i=1,2)$ and $\psi^{\prime}: \mathbb{Z}\left[H_{1}(Y)\right] \rightarrow R$ be ring homomorphisms induced by $\psi$. Then we have

$$
\tau^{\psi}(X) \cdot \tau^{\psi^{\prime}}(Y) \doteq \tau^{\psi_{1}}\left(X_{1}\right) \cdot \tau^{\psi_{2}}\left(X_{2}\right)
$$

Lemma 2.2 (1) Let $t$ be a generator of $H_{1}\left(S^{1} \times D^{2}\right)$. Then we have

$$
\tau\left(S^{1} \times D^{2}\right) \doteq(t-1)^{-1} .
$$

(2) $\tau\left(S^{1} \times S^{1}\right) \doteq 1$.

(3) Let $L=K_{1} \cup \cdots \cup K_{n}$ be an $n$-component link in a homology sphere, $E$ the exterior of $L$, and $t_{i}(i=1, \cdots, n)$ the homology class of a meridian of $K_{i}$ in $H_{1}(E)$. Then we have

$$
\tau(E) \doteq \begin{cases}\Delta_{L}\left(t_{1}\right)\left(t_{1}-1\right)^{-1} & (n=1), \\ \Delta_{L}\left(t_{1}, \cdots, t_{n}\right) & (n \geq 2) .\end{cases}
$$

Lemma 2.3 Let $X$ be a finite $C W$-complex, and $\psi: \mathbb{Z}\left[H_{1}(X)\right] \rightarrow R$ and $\psi^{\prime}: R \rightarrow R^{\prime}$ ring homomorphisms. If $\tau^{\psi}(X) \neq 0$ and $\psi^{\prime}\left(\tau^{\psi}(X)\right) \neq 0$, then we have

$$
\tau^{\psi^{\prime} \circ \psi}(X) \doteq \psi^{\prime}\left(\tau^{\psi}(X)\right) \text {. }
$$

Lemma 2.4 Let $H$ be a finitely generated abelian group. If $h$ is an element of $H$ with infinite order, then $h-1$ is invertible in $Q(\mathbb{Z}[H])$. 
Let $L=K_{1} \cup \cdots \cup K_{n}$ be an $n$-component link in a homology sphere $\Sigma$. Let $p_{i}$ and $q_{i}(i=1, \cdots, n)$ be integers satisfying $p_{i} \geq 0, q_{i} \neq 0$ and $\operatorname{gcd}\left(p_{i}, q_{i}\right)=1$ for each $i$. Then $\Sigma\left(L ; p_{1} / q_{1}, \cdots, p_{n} / q_{n}\right)$ is defined as follows:

(1) Let $N(L)$ be a regular neighborhood of $L$ in $\Sigma$. Note that $N(L)$ is a disjoint union of $N\left(K_{i}\right)$ 's ; $N(L)=\bigcup_{i=1}^{n} N\left(K_{i}\right)$, where $N\left(K_{i}\right)$ is a regular neighborhood of $K_{i}$. Let $E_{L}$ denote the exterior of $L: E_{L}=\overline{\Sigma \backslash N(L)}$. Note that $\partial E_{L}=\bigcup_{i=1}^{n} \partial N\left(K_{i}\right)$.

(2) Let $m_{i}$ and $l_{i}(i=1, \cdots, n)$ be a meridian and a longitude of $K_{i}$, which lie on $\partial N\left(K_{i}\right) \subset \partial E_{L}$. We take a simple closed curve $m_{i}^{\prime}$ on $\partial N\left(K_{i}\right)(i=1, \cdots, n)$ such that $\left[m_{i}^{\prime}\right]=\left[m_{i}\right]^{p_{i}}\left[l_{i}\right]^{q_{i}}$ in $H_{1}\left(\partial N\left(K_{i}\right)\right)$. Let $r_{i}$ and $s_{i}(i=1, \cdots, n)$ be integers satisfying $p_{i} s_{i}-q_{i} r_{i}=1$. We take a simple closed curve $l_{i}^{\prime}$ on $\partial N\left(K_{i}\right)(i=1, \cdots, n)$ such that $\left[l_{i}^{\prime}\right]=\left[m_{i}\right]^{r_{i}}\left[l_{i}\right]^{s_{i}}$ in $H_{1}\left(\partial N\left(K_{i}\right)\right)$.

(3) Let $V_{1}, \cdots, V_{n}$ be $n$-copies of $S^{1} \times D^{2}$. Then $\Sigma\left(L ; p_{1} / q_{1}, \cdots, p_{n} / q_{n}\right)$ is defined as the 3-manifold obtained from $E_{L}$ and $\left\{V_{1}, \cdots, V_{n}\right\}$ by identifying $\partial N\left(K_{i}\right)$ and $\partial V_{i}$ by a homeomorphism such that $m_{i}^{\prime}$ is identified with a meridian of $V_{i}$ for each $i$. Note that $l_{i}^{\prime}$ is identified with a longitude of $V_{i}$. We sometimes express

$$
\Sigma\left(L ; p_{1} / q_{1}, \cdots, p_{n} / q_{n}\right)=E_{L} \cup V_{1} \cup \cdots \cup V_{n}
$$

schematically.

(4) Let $M=\Sigma\left(L ; p_{1} / q_{1}, \cdots, p_{n} / q_{n}\right)$ as above. We define $M_{k}(k=1, \cdots, n)$ by

$$
M_{k}=\overline{M \backslash\left(V_{1} \cup \cdots \cup V_{k}\right)}=E_{L} \cup V_{k+1} \cup \cdots \cup V_{n} .
$$

Note that $M \supset M_{1} \supset \cdots \supset M_{n}=E_{L}$.

We assume that $M$ is a homology lens space with $H_{1}(M) \cong \mathbb{Z} / p \mathbb{Z}(p \geq 2)$. Let $T$ be a generator of $H_{1}(M), d \geq 2$ a divisor of $p$ and $\psi_{d}: \mathbb{Z}\left[H_{1}(M)\right] \rightarrow \mathbb{Q}\left(\zeta_{d}\right)$ a ring homomorphism such that $\psi_{d}(T)=\zeta_{d}$. We then define $\psi_{k, d}: \mathbb{Z}\left[H_{1}\left(M_{k}\right)\right] \rightarrow \mathbb{Q}\left(\zeta_{d}\right)$ by $\psi_{k, d}=\psi_{d} \circ \iota_{k}$, where $\iota_{k}: \mathbb{Z}\left[H_{1}\left(M_{k}\right)\right] \rightarrow \mathbb{Z}\left[H_{1}(M)\right]$ is a ring homomorphism induced from the natural inclusion $M_{k} \hookrightarrow M$. Then we have the following surgery formula for the Reidemeister torsion.

Lemma 2.5 (1) If $n \geq 2$ and $\psi_{d}\left(\left[l_{i}^{\prime}\right]\right) \neq 1(i=1, \cdots, n)$, then we have

$$
\tau^{\psi_{d}}(M) \doteq \Delta_{L}\left(\xi_{1}, \cdots, \xi_{n}\right) \prod_{i=1}^{n}\left(\psi_{d}\left(\left[l_{i}^{\prime}\right]\right)-1\right)^{-1},
$$

where $\xi_{i}=\psi_{d}\left(\left[m_{i}\right]\right)$ 
(2) If $n \geq 2$, and $\left[l_{i}^{\prime}\right](i=k+1, \cdots, n)$ has infinite order in $H_{1}\left(M_{k}\right)$, then we have

$$
\tau\left(M_{k}\right) \doteq \Delta_{L}\left(\left[m_{1}\right], \cdots,\left[m_{n}\right]\right) \prod_{i=k+1}^{n}\left(\left[l_{i}^{\prime}\right]-1\right)^{-1} \quad \text { in } Q\left(\mathbb{Z}\left[H_{1}\left(M_{k}\right)\right]\right) .
$$

(3) If $n \geq 1, \tau\left(M_{k}\right) \neq 0$ and $\psi_{d}\left(\left[l_{i}^{\prime}\right]\right) \neq 1(i=1, \cdots, k)$, then we have

$$
\tau^{\psi_{d}}(M) \doteq \psi_{k, d}\left(\tau\left(M_{k}\right)\right) \prod_{i=1}^{k}\left(\psi_{d}\left(\left[l_{i}^{\prime}\right]\right)-1\right)^{-1}
$$

The following is an explicit form of Lemma 2.5 (3) in the case $n=k=1$.

Lemma 2.6 Let $K$ be a knot in a homology sphere $\Sigma$ and $M=\Sigma(K ; p / q)$ $(p \geq 2)$. Let $T$ be a generator of $H_{1}(M)$ that corresponds to a meridian of $K$, and $\psi_{d}: \mathbb{Z}\left[H_{1}(M)\right] \rightarrow \mathbb{Q}\left(\zeta_{d}\right)$ a ring homomorphism such that $\psi_{d}(T)=\zeta_{d}$. Then we have

$$
\tau^{\psi_{d}}(M) \doteq \Delta_{K}\left(\zeta_{d}\right)\left(\zeta_{d}-1\right)^{-1}\left(\zeta_{d}^{\bar{q}}-1\right)^{-1}
$$

where $\bar{q}$ is the inverse element of $q$ in $(\mathbb{Z} / d \mathbb{Z})^{\times}$.

\section{Conditions from the first homology group}

We consider constraints on the multiplicities of singular fibers that come from the assumption that the first homology group is finite cyclic. Let $M$ be a Seifert fibered homology lens space as in the setting in Section 1:

$$
M=S^{3}\left(L ; p_{1} / q_{1}, \cdots, p_{N} / q_{N}, a\right)=E_{L} \cup V_{1} \cup \cdots \cup V_{N} \cup V_{N+1} .
$$

We take $m_{i}$ and $l_{i}(i=1, \cdots, N+1)$ on $\partial E_{L}$ as in Section 2. We also take $m_{i}^{\prime}$ and $l_{i}^{\prime}$ on $\partial E_{L}$ as in Section 2 except $m_{N+1}^{\prime}$ and $l_{N+1}^{\prime}$ such that

$$
\left[m_{N+1}^{\prime}\right]=\left[m_{N+1}\right]^{a}\left[l_{N+1}\right] \text { and }\left[l_{N+1}^{\prime}\right]=\left[m_{N+1}\right] .
$$

Then we have the following:

Lemma 3.1 The first homology group $H_{1}(M)$ is generated by $\left[m_{i}\right],\left[l_{i}\right],\left[m_{i}^{\prime}\right]$ and $\left[l_{i}^{\prime}\right](i=1, \cdots, N+1)$ subject to the following relations:

$$
\begin{aligned}
{\left[m_{i}^{\prime}\right] } & =\left[m_{i}\right]^{p_{i}}\left[l_{i}\right]^{q_{i}}=1(i=1, \cdots, N), & {\left[m_{N+1}^{\prime}\right] } & =\left[m_{N+1}\right]^{a}\left[l_{N+1}\right]=1, \\
{\left[l_{i}\right] } & =\left[m_{N+1}\right](i=1, \cdots, N), & {\left[m_{1}\right] \cdots\left[m_{N}\right] } & =\left[l_{N+1}\right], \\
{\left[l_{i}^{\prime}\right] } & =\left[m_{i}\right]^{r_{i}}\left[l_{i}\right]^{s_{i}}(i=1, \cdots, N), & {\left[l_{N+1}^{\prime}\right] } & =\left[m_{N+1}\right] .
\end{aligned}
$$


The presentation matrix $A$ of $H_{1}(M)$ with respect to $\left[m_{1}\right], \cdots,\left[m_{N+1}\right]$ is

$$
A=\left(\begin{array}{cccc}
p_{1} & & 0 & q_{1} \\
& \ddots & & \vdots \\
0 & & p_{N} & q_{N} \\
1 & \cdots & 1 & a
\end{array}\right) .
$$

Here the operation is additive. We recall $H_{1}(M) \cong \mathbb{Z} / p \mathbb{Z}$.

Lemma 3.2 Let $\tau=p_{1} \cdots p_{N}$ and $\tau_{i}=\tau / p_{i}(i=1, \cdots, N)$. Then

$$
p=\left|\tau a-\sum_{i=1}^{N} q_{i} \tau_{i}\right| .
$$

In particular, if $d$ divides $p_{i}$ and $p_{j}(i \neq j)$, then $d$ divides $p$.

Proof It is well known that $|\operatorname{det} A|$ is the order of $H_{1}(M)$ and

$$
\operatorname{det} A=\tau a-\sum_{i=1}^{N} q_{i} \tau_{i}
$$

(cf Saviliev [16]). Since the order of $H_{1}(M)$ is $p$, we have the result.

Lemma 3.3 Let $A_{i, j}$ be the cofactor of $(i, j)$-entry of $A$. Then we have

$$
\begin{aligned}
A_{i, N+1} & = \pm \tau_{i} & & (i=1, \cdots, N), \\
A_{i, i} & =\tau_{i} a-\sum_{j \neq i} q_{j} \tau_{i j} & & (i=1, \cdots, N), \\
A_{i, j} & = \pm q_{j} \tau_{i j} & & (i \neq j), \\
A_{N+1, i} & = \pm q_{i} \tau_{i} & & (i=1, \cdots, N), \\
A_{N+1, N+1} & =\tau, & &
\end{aligned}
$$

where $\tau_{i j}=\tau / p_{i} p_{j}(i \neq j)$.

The following is a key to prove results stated in Section 1 .

Lemma 3.4 (1) Let $d \geq 2$ be a common divisor of $p_{i}$ and $p_{j}(i \neq j)$. Then we have $\operatorname{gcd}\left(d, p_{k}\right)=1$ for $k \neq i, j$.

(2) Let $\ell$ be a prime divisor of $\operatorname{gcd}\left(p, p_{i}\right)$. Then there exists $j \neq i$ such that $\ell$ is a divisor of $p_{j}$.

(3) If $\operatorname{gcd}\left(p_{i}, p_{j}\right)=1$ for every pair $\{i, j\}$, then we have $\operatorname{gcd}\left(p, p_{i}\right)=1$ for every $i$. 
Proof (1) Let $\delta$ be the greatest common divisor of all $N$-minors of $A$. Since $H_{1}(M)$ is cyclic, we have $\delta=1$ by the elementary divisor theory. Suppose that there exists $k \neq i, j$ such that $\operatorname{gcd}\left(d, p_{k}\right) \geq 2$. Then each $A_{i, j}$ is divisible by $\operatorname{gcd}\left(d, p_{k}\right)$ by Lemma 3.3, and hence $\delta$ is divisible by $\operatorname{gcd}\left(d, p_{k}\right)$. This contradicts $\delta=1$.

(2) By Lemma 3.2 and $\operatorname{gcd}\left(p_{i}, q_{i}\right)=1, \ell$ divides $\tau_{i}$. Hence there exists $j \neq i$ such that $\ell$ divides $p_{j}$.

(3) Suppose there exists $i$ such that $\operatorname{gcd}\left(p, p_{i}\right) \geq 2$. We take a prime divisor $\ell$ of $\operatorname{gcd}\left(p, p_{i}\right)$. Then there exists $j \neq i$ such that $\ell$ divides $p_{j}$ by (2). This contradicts $\operatorname{gcd}\left(p_{i}, p_{j}\right)=1$.

Remark 3.5 We can show the following, although not needed here.

(1) $H_{1}(M)$ is cyclic if and only if $\delta=1$.

(2) $\delta$ coincides with the greatest common divisor of $\tau_{i j}$ 's.

\section{Proofs of Theorem 1.1, Theorem 1.2 and Theorem 1.3}

We need the following well known fact (cf Nicolaescu [13]).

Lemma 4.1 Let $L$ be the link in Figure 1. Then we have

$$
\Delta_{L}\left(t_{1}, \cdots, t_{N}, t_{N+1}\right)=\left(t_{N+1}-1\right)^{N-1} .
$$

Recall that

$$
M=S^{3}\left(L ; p_{1} / q_{1}, \cdots, p_{N} / q_{N}, a\right)=E_{L} \cup V_{1} \cup \cdots \cup V_{N+1} .
$$

When a generator $T$ of $H_{1}(M)$ and a ring homomorphism $\psi_{d}: \mathbb{Z}\left[H_{1}(M)\right] \rightarrow \mathbb{Q}\left(\zeta_{d}\right)$ such that $\psi_{d}(T)=\zeta_{d}$ are given, we set $\xi_{i}=\psi_{d}\left(\left[m_{i}\right]\right)(i=1, \cdots, N+1)$. Then $\xi_{i}$ is a $d$-th root of unity and $\left\{\xi_{1}, \cdots, \xi_{N+1}\right\}$ generates $\psi_{d}\left(H_{1}(M)\right) \cong \mathbb{Z} / d \mathbb{Z}$. The next lemma follows from Lemma 3.1.

Lemma 4.2 The following relations hold:

(1) $\xi_{i}^{p_{i}} \xi_{N+1}^{q_{i}}=1(i=1, \cdots, N)$,

(2) $\left(\prod_{i=1}^{N} \xi_{i}\right) \xi_{N+1}^{a}=1$,

(3) $\psi_{d}\left(\left[l_{i}^{\prime}\right]\right)=\xi_{i}^{r_{i}} \xi_{N+1}^{s_{i}}(i=1, \cdots, N) \quad$ and $\quad \psi_{d}\left(\left[l_{N+1}^{\prime}\right]\right)=\xi_{N+1}$. 
Proof of Theorem 1.1 (1) Let $d \geq 2$ be as in the statement of Theorem 1.1. By Lemma $4.2(1)$ and $\operatorname{gcd}\left(d, p_{i}\right)=1(i=1, \cdots, N)$, we have $\xi_{i}=\xi_{N+1}^{-q_{i} \bar{p}_{i}}$, where $\bar{p}_{i}$ is the inverse element of $p_{i}$ in $(\mathbb{Z} / d \mathbb{Z})^{\times}$. Hence $\xi_{N+1}$ generates $\psi_{d}\left(H_{1}(M)\right) \cong \mathbb{Z} / d \mathbb{Z}$. Since a generator of $\psi_{d}\left(H_{1}(M)\right)$ is a primitive $d$-th root of unity, $\xi_{N+1}=\zeta_{d}^{u}$ is also a primitive $d$-th root of unity. Therefore we have $\operatorname{gcd}(d, u)=1$.

By Lemma $4.2(3), \xi_{i}=\xi_{N+1}^{-q_{i} \bar{p}_{i}}$ and $p_{i} s_{i}-q_{i} r_{i}=1$, we have

$$
\psi_{d}\left(\left[l_{i}^{\prime}\right]\right)=\xi_{i}^{r_{i}} \xi_{N+1}^{s_{i}}=\xi_{N+1}^{-q_{i} r_{i} \bar{p}_{i}+s_{i}}=\xi_{N+1}^{\bar{p}_{i}\left(p_{i} s_{i}-q_{i} r_{i}\right)}=\xi_{N+1}^{\bar{p}_{i}}(i=1, \cdots, N)
$$

and $\psi_{d}\left(\left[l_{N+1}^{\prime}\right]\right)=\xi_{N+1}$. Hence $\psi_{d}\left(\left[l_{i}^{\prime}\right]\right)(i=1, \cdots, N+1)$ is a primitive $d$-th root of unity.

By Lemma 2.5 (1) and Lemma 4.1, we have

$$
\begin{aligned}
\tau^{\psi_{d}}(M) & \doteq \Delta_{L}\left(\xi_{1}, \cdots, \xi_{N+1}\right) \prod_{i=1}^{N+1}\left(\psi_{d}\left(\left[l_{i}^{\prime}\right]\right)-1\right)^{-1} \\
& =\left(\xi_{N+1}-1\right)^{N-1}\left(\xi_{N+1}-1\right)^{-1} \prod_{i=1}^{N}\left(\xi_{N+1}^{\bar{p}_{i}}-1\right)^{-1} \\
& =\left(\zeta_{d}^{u}-1\right)^{N-2} \prod_{i=1}^{N}\left(\xi_{d}^{u \bar{p}_{i}}-1\right)^{-1} .
\end{aligned}
$$

(2) Let $M$ be the result of $p / q$-surgery along a knot $K$ in a homology sphere $\Sigma$ : $M=\Sigma(K ; p / q)$. We take the homology class of a meridian for $K$ as $T$. By (1) and Lemma 2.6, we have

$$
\Delta_{K}\left(\zeta_{d}\right)\left(\zeta_{d}-1\right)^{-1}\left(\zeta_{d}^{\bar{q}}-1\right)^{-1} \doteq\left(\zeta_{d}^{u}-1\right)^{N-2} \prod_{i=1}^{N}\left(\zeta_{d}^{u \bar{p}_{i}}-1\right)^{-1}
$$

By noting $\operatorname{gcd}(d, q)=\operatorname{gcd}(d, u)=\operatorname{gcd}\left(d, p_{i}\right)=1$, we have

$$
\left|N_{d}\left(\zeta_{d}-1\right)\right|=\left|N_{d}\left(\zeta_{d}^{\bar{q}}-1\right)\right|=\left|N_{d}\left(\zeta_{d}^{u}-1\right)\right|=\left|N_{d}\left(\zeta_{d}^{u \bar{p}_{i}}-1\right)\right| \neq 0 .
$$

Therefore by taking the norms of both sides of (4-1), we have $\left|N_{d}\left(\Delta_{K}\left(\zeta_{d}\right)\right)\right|=1$ and hence $|M|_{d}=1$.

(3) If $d^{\prime}$ divides $d$, then we have $\operatorname{gcd}\left(d^{\prime}, p_{i}\right)=1$ for all $i$. Hence $|M|_{d^{\prime}}=1$ by (2). Thus we have

$$
\|M\|_{d}=\prod_{d^{\prime} \mid d}|M|_{d^{\prime}}=1
$$

Algebraic $8 \mathcal{G}$ Geometric Topology, Volume 7 (2007) 
Lemma 4.3 For $1 \leq j<i \leq N$, let $M_{j}=E_{L} \cup V_{j+1} \cup \cdots \cup V_{N+1}$ be a 3-manifold as in Section 2. Then we have

$$
\left(\left[m_{N+1}\right]-1\right)\left(\left[l_{i}^{\prime}\right]-1\right)^{-1}=\sum_{k=0}^{p_{i}-1}\left[l_{i}^{\prime}\right]^{k} \text { in } Q\left(\mathbb{Z}\left[H_{1}\left(M_{j}\right)\right]\right) .
$$

Proof We prove the lemma in steps.

Step $1\left[m_{N+1}\right]$ in $H_{1}\left(M_{j}\right)$ has infinite order.

Proof In this step, we write operation additively, and consider $H_{1}\left(M_{j} ; \mathbb{Q}\right)$ as a vector space over $\mathbb{Q}$. It is sufficient to prove $\left[m_{N+1}\right] \neq 0$ in $H_{1}\left(M_{j} ; \mathbb{Q}\right)$.

Since $M$ is a rational homology sphere and $M_{j}$ is the exterior of a $j$-component link in $M$ with $\left\{m_{1}^{\prime}, \cdots, m_{j}^{\prime}\right\}$ as meridians, we have $\operatorname{dim} H_{1}\left(M_{j} ; \mathbb{Q}\right)=j$ and that $\left\{\left[m_{1}^{\prime}\right], \cdots,\left[m_{j}^{\prime}\right]\right\}$ is a basis.

Assume that $\left[m_{N+1}\right]=0$ in $H_{1}\left(M_{j} ; \mathbb{Q}\right)$. Then by the relations $\left[l_{i}\right]=\left[m_{N+1}\right](i=$ $1, \cdots, N)$ etc, we have $\left[m_{1}^{\prime}\right]=p_{1}\left[m_{1}\right], \cdots,\left[m_{j}^{\prime}\right]=p_{j}\left[m_{j}\right]$ and $\left[m_{1}\right]+\cdots+\left[m_{j}\right]=0$. This contradicts the fact that $\left\{\left[m_{1}^{\prime}\right], \cdots,\left[m_{j}^{\prime}\right]\right\}$ is a basis.

Step $2\left[m_{N+1}\right]=\left[l_{i}^{\prime}\right]^{p_{i}}(i>j)$ in $H_{1}\left(M_{j}\right)$.

Proof By the relations

$$
\left[m_{i}\right]^{p_{i}}\left[m_{N+1}\right]^{q_{i}}=1,\left[m_{i}\right]^{r_{i}}\left[m_{N+1}\right]^{s_{i}}=\left[l_{i}^{\prime}\right] \text { and } p_{i} s_{i}-q_{i} r_{i}=1
$$

that hold in $H_{1}\left(M_{j}\right)$ for $i \geq j+1$, we have

$$
\left[m_{N+1}\right]=\left[m_{N+1}\right]^{p_{i} s_{i}-q_{i} r_{i}}=\left[m_{N+1}\right]^{p_{i} s_{i}}\left[m_{i}\right]^{p_{i} r_{i}}=\left(\left[m_{i}\right]^{r_{i}}\left[m_{N+1}\right]^{s_{i}}\right)^{p_{i}}=\left[l_{i}^{\prime}\right]^{p_{i}} \text {. }
$$

Step $3\left[l_{i}^{\prime}\right](i>j)$ in $H_{1}\left(M_{j}\right)$ has infinite order.

Proof If there exists an index $i(j+1 \leq i \leq N)$ such that $\left[l_{i}^{\prime}\right]$ has finite order, then $\left[m_{N+1}\right]$ has finite order by Step 2. This contradicts Step 1 .

By Step 3 and Lemma 2.4, $\left[l_{i}^{\prime}\right]-1$ is invertible in $Q\left(\mathbb{Z}\left[H_{1}\left(M_{j}\right)\right]\right)$ for $i>j$. Hence we have the result by Step 2.

Proof of Theorem 1.2 (1) By Lemma 3.2, $d$ divides $p$. By Lemma 3.4 (1), $\operatorname{gcd}\left(d, p_{i}\right)=1(i=3, \cdots, N)$. Since $\operatorname{gcd}\left(p_{1}, q_{1}\right)=1$ and $d$ divides $p_{1}$, we have $\operatorname{gcd}\left(d, q_{1}\right)=1$. Similarly we have $\operatorname{gcd}\left(d, q_{2}\right)=1$.

(2) We prove this part in steps.

Step $1 \xi_{2}=\xi_{1}^{-1}$ and $\xi_{i}=1(i=3, \cdots, N+1)$. 
Proof Since $\xi_{1}^{d}=1$ and $d$ divides $p_{1}$, we have $\xi_{1}^{p_{1}}=1$. Since $\xi_{1}^{p_{1}}=1, \operatorname{gcd}\left(d, q_{1}\right)=$ 1 and $\xi_{1}^{p_{1}} \xi_{N+1}^{q_{1}}=1$ in Lemma $4.2(1)$, we have $\xi_{N+1}=1$. Since $\xi_{N+1}=1$, $\operatorname{gcd}\left(d, p_{i}\right)=1(i=3, \cdots, N)$ and $\xi_{i}^{p_{i}} \xi_{N+1}^{q_{i}}=1$, we have $\xi_{i}=1(i=3, \cdots, N)$, and hence $\xi_{1} \xi_{2}=1$ by Lemma $4.2(2)$.

Step $2 \xi_{1}=\zeta_{d}^{u_{1}}, \xi_{2}=\zeta_{d}^{-u_{1}}$ and $\operatorname{gcd}\left(d, u_{1}\right)=1$

Proof We have $\xi_{1}=\psi_{d}\left(\left[m_{1}\right]\right)=\psi_{d}\left(T^{u_{1}}\right)=\zeta_{d}^{u_{1}}$. By Step $1, \xi_{2}=\xi_{1}^{-1}=\zeta_{d}^{-u_{1}}$, and $\xi_{1}=\zeta_{d}^{u_{1}}$ generates $\psi_{d}\left(H_{1}(M)\right) \cong \mathbb{Z} / d \mathbb{Z}$. Hence we have $\operatorname{gcd}\left(d, u_{1}\right)=1$.

We set $M_{2}=E_{L} \cup V_{3} \cup \cdots \cup V_{N+1}$ as in Section 2 .

Step $3 \tau\left(M_{2}\right) \doteq \prod_{i=3}^{N}\left(\sum_{k=0}^{p_{i}-1}\left[l_{i}^{\prime}\right]^{k}\right)$ in $Q\left(\mathbb{Z}\left[H_{1}\left(M_{2}\right)\right]\right)$.

Proof By Lemma 2.5 (2), Lemma 4.1 and Lemma 4.3, we have

$$
\tau\left(M_{2}\right) \doteq\left(\left[m_{N+1}\right]-1\right)^{N-2} \prod_{i=3}^{N}\left(\left[l_{i}^{\prime}\right]-1\right)^{-1}=\prod_{i=3}^{N}\left(\sum_{k=0}^{p_{i}-1}\left[l_{i}^{\prime}\right]^{k}\right) .
$$

By Lemma 4.2 (3), Step 1 and Step 2, we have $\psi_{d}\left(\left[l_{i}^{\prime}\right]\right)=1(i \geq 3), \psi_{d}\left(\left[l_{1}^{\prime}\right]\right)=\zeta_{d}^{-u_{1} \bar{q}_{1}}$ and $\psi_{d}\left(\left[l_{2}^{\prime}\right]\right)=\zeta_{d}^{u_{1} \bar{q}_{2}}$. Hence we have the result by Lemma 2.5 (3) and Step 3.

(3) Let $M$ be the result of $p / q$-surgery along a knot $K$ in a homology sphere $\Sigma$. We take the homology class of a meridian for $K$ in $H_{1}(M)$ as $T$. By (2) and Lemma 2.6, we have

$$
\Delta_{K}\left(\zeta_{d}\right)\left(\zeta_{d}-1\right)^{-1}\left(\zeta_{d}^{\bar{q}}-1\right)^{-1} \doteq p_{3} \cdots p_{N}\left(\zeta_{d}^{u_{1} \bar{q}_{1}}-1\right)^{-1}\left(\zeta_{d}^{u_{1} \bar{q}_{2}}-1\right)^{-1}
$$

By noting $\operatorname{gcd}(d, q)=\operatorname{gcd}\left(d, u_{1}\right)=\operatorname{gcd}\left(d, q_{1}\right)=\operatorname{gcd}\left(d, q_{2}\right)=1$, we have

$$
\left|N_{d}\left(\zeta_{d}-1\right)\right|=\left|N_{d}\left(\zeta_{d}^{\bar{q}}-1\right)\right|=\left|N_{d}\left(\zeta_{d}^{u_{1} \bar{q}_{1}}-1\right)\right|=\left|N_{d}\left(\zeta_{d}^{u_{1} \bar{q}_{2}}-1\right)\right| \neq 0 .
$$

By taking the norms of both sides of (4-2), we obtain $|M|_{d}=\left|N_{d}\left(\Delta_{K}\left(\zeta_{d}\right)\right)\right|=$ $\left(p_{3} \cdots p_{N}\right)^{\varphi(d)}$.

Proof of Theorem 1.3 It is sufficient to prove (1) and (3).

(1) Suppose $\|M\|_{p}=1$. By Theorem $1.2(2)$, we have $\operatorname{gcd}\left(p_{i}, p_{j}\right)=1$ for every pair $\{i, j\}$.

Suppose $\operatorname{gcd}\left(p_{i}, p_{j}\right)=1$ for every pair $\{i, j\}$. By Lemma 3.4 (3), we have that $\operatorname{gcd}\left(p, p_{i}\right)=1$ for every $i$. 
Suppose $\operatorname{gcd}\left(p, p_{i}\right)=1$ for every $i=1, \cdots, N$. By Theorem 1.1 (3), we have $\|M\|_{p}=1$.

(3) Let $M$ be the result of $p / q$-surgery along a knot $K$ in a homology sphere $\Sigma$. We first prove "if" part. Without loss of generality, we may assume that $\{i, j\}=\{1,2\}$, and $\{k, h\}=\{1,3\}$ or $\{3,4\}$. Hence it is sufficient to prove Step 1 and Step 2 below.

Step 1 If $\operatorname{gcd}\left(p_{1}, p_{2}\right) \geq 2$ and $\operatorname{gcd}\left(p_{1}, p_{3}\right) \geq 2$, then there exists a divisor $d \geq 2$ of $p$ such that $\Delta_{K}\left(\zeta_{d}\right)=0$.

Proof Take a divisor $d_{2} \geq 2$ of $\operatorname{gcd}\left(p_{1}, p_{2}\right)$ and a divisor $d_{3} \geq 2$ of $\operatorname{gcd}\left(p_{1}, p_{3}\right)$, and set $d=d_{2} d_{3}$. By Lemma 3.4 (1), we have $\operatorname{gcd}\left(d_{2}, d_{3}\right)=1$, and hence $d$ is a divisor of $p_{1}$. By Lemma 3.2, both $d_{2}$ and $d_{3}$ are divisors of $p$, and hence $d$ is a divisor of $p$ because $\operatorname{gcd}\left(d_{2}, d_{3}\right)=1$.

Let $\psi_{d}: \mathbb{Z}\left[H_{1}(M)\right] \rightarrow \mathbb{Q}\left(\zeta_{d}\right)$ be a ring homomorphism such that $\psi_{d}(T)=\zeta_{d}$, where $T$ is a generator of $H_{1}(M)$ that corresponds to a meridian of $K$. We set $\psi_{d}\left(\left[m_{i}\right]\right)=$ $\xi_{i}(i=1, \cdots, N+1)$, where $m_{i}$ is a meridian of $K_{i}$. Since $d$ is a divisor of $p_{1}$, $\operatorname{gcd}\left(d, q_{1}\right)=1$ and $\xi_{1}^{p_{1}} \xi_{N+1}^{q_{1}}=1$ in Lemma 4.2 (1), we have $\xi_{N+1}=1$. Hence $\xi_{i}^{p_{i}}=1$ for all $i$ by Lemma 4.2 (1). By Lemma $3.4(1), \operatorname{gcd}\left(d_{2}, p_{i}\right)=1$ for $i \neq 1,2$, and $\operatorname{gcd}\left(d_{3}, p_{i}\right)=1$ for $i \neq 1,3$. Hence $\operatorname{gcd}\left(d, p_{i}\right)=1$ for $i \geq 4$. Since $\xi_{i}^{p_{i}}=1$, we have $\xi_{i}=1$ for $i \geq 4$, and $\xi_{1} \xi_{2} \xi_{3}=1$ by Lemma 4.2 (2). Therefore $\psi_{d}\left(H_{1}(M)\right)$ is generated by $\xi_{2}$ and $\xi_{3}$. We note that $\operatorname{gcd}\left(d_{2}, p_{3}\right)=\operatorname{gcd}\left(d_{3}, p_{2}\right)=1$ are used in the next paragraph.

Since $\xi_{2}^{p_{2}}=1$, we have $\left(\xi_{2}^{d_{2}}\right)^{p_{2}}=1$. On the other hand $\left(\xi_{2}^{d_{2}}\right)^{d_{3}}=\xi_{2}^{d}=1$. Hence $\xi_{2}^{d_{2}}=1$ since $\operatorname{gcd}\left(d_{3}, p_{2}\right)=1$. Similarly $\xi_{3}^{d_{3}}=1$ since $\operatorname{gcd}\left(d_{2}, p_{3}\right)=1$. If one of $\xi_{2}$ and $\xi_{3}$ is not "primitive", then $\xi_{2}$ and $\xi_{3}$ do not generate $\psi_{d}\left(H_{1}(M)\right)$. Hence $\xi_{2}$ and $\xi_{3}$ are primitive $d_{2}$-th and $d_{3}$-th root of unities respectively, and hence $\xi_{1}$ is a primitive $d$-th root of unity since $\xi_{1} \xi_{2} \xi_{3}=1$ and $\operatorname{gcd}\left(d_{2}, d_{3}\right)=1$. Since $\operatorname{gcd}\left(p_{1}, q_{1}\right)=$ $\operatorname{gcd}\left(p_{2}, q_{2}\right)=1$ and $d_{2}$ divides $p_{1}$ and $p_{2}$, we have $\operatorname{gcd}\left(d_{2}, q_{1}\right)=\operatorname{gcd}\left(d_{2}, q_{2}\right)=1$. Similarly we have $\operatorname{gcd}\left(d_{3}, q_{1}\right)=\operatorname{gcd}\left(d_{3}, q_{3}\right)=1$. Since $\operatorname{gcd}\left(d_{2}, q_{1}\right)=\operatorname{gcd}\left(d_{3}, q_{1}\right)=1$, we have $\operatorname{gcd}\left(d, q_{1}\right)=1$. Hence we have

$$
\psi_{d}\left(\left[l_{i}^{\prime}\right]\right)=\xi_{i}^{-\bar{q}_{i}} \neq 1 \quad(i=1,2,3),
$$

where $\bar{q}_{1}, \bar{q}_{2}$ and $\bar{q}_{3}$ are integers satisfying $q_{1} \bar{q}_{1} \equiv 1(\bmod d), q_{2} \bar{q}_{2} \equiv 1\left(\bmod d_{2}\right)$ and $q_{3} \bar{q}_{3} \equiv 1\left(\bmod d_{3}\right)$ respectively.

We set $M_{3}=E_{L} \cup V_{4} \cup \cdots \cup V_{N+1}$ and $\psi_{3, d}: \mathbb{Z}\left[H_{1}\left(M_{3}\right)\right] \rightarrow \mathbb{Q}\left(\zeta_{d}\right)$ as in Section 2. By Lemma 4.3, we have

$$
\tau\left(M_{3}\right) \doteq\left(\left[m_{N+1}\right]-1\right)^{N-2} \prod_{i=4}^{N}\left(\left[l_{i}^{\prime}\right]-1\right)^{-1}=\left(\left[m_{N+1}\right]-1\right) \prod_{i=4}^{N}\left(\sum_{k=0}^{p_{i}-1}\left[l_{i}^{\prime}\right]^{k}\right),
$$


and hence $\tau^{\psi_{3, d}}\left(M_{3}\right)=0$. By Lemma 2.5 (3), we have

$$
\tau^{\psi_{d}}(M) \doteq \tau^{\psi_{3, d}}\left(M_{3}\right) \prod_{i=1}^{3}\left(\xi_{i}^{\bar{q}_{i}}-1\right)^{-1}=0,
$$

and hence $\Delta_{K}\left(\zeta_{d}\right)=0$ by Lemma 2.6.

Step 2 If $\operatorname{gcd}\left(p_{1}, p_{2}\right) \geq 2$ and $\operatorname{gcd}\left(p_{3}, p_{4}\right) \geq 2(N \geq 4)$, then there exists a divisor $d \geq 2$ of $p$ such that $\Delta_{K}\left(\zeta_{d}\right)=0$.

Proof Take a divisor $d_{1} \geq 2$ of $\operatorname{gcd}\left(p_{1}, p_{2}\right)$ and a divisor $d_{3} \geq 2$ of $\operatorname{gcd}\left(p_{3}, p_{4}\right)$, and set $d=d_{1} d_{3}$. By Lemma $3.4(1)$, we have $\operatorname{gcd}\left(d_{1}, d_{3}\right)=1$. By Lemma 3.2, both $d_{1}$ and $d_{3}$ are divisors of $p$. Hence $d$ is a divisor of $p$ since $\operatorname{gcd}\left(d_{1}, d_{3}\right)=1$.

Let $\psi_{d}: \mathbb{Z}\left[H_{1}(M)\right] \rightarrow \mathbb{Q}\left(\zeta_{d}\right)$ be a ring homomorphism such that $\psi_{d}(T)=\zeta_{d}$, where $T$ is a generator of $H_{1}(M)$ that corresponds to a meridian of $K$. We set $\psi_{d}\left(\left[m_{i}\right]\right)=$ $\xi_{i}(i=1, \cdots, N+1)$, where $m_{i}$ is a meridian of $K_{i}$. Since $d_{1}$ divides $p_{1}$, we have $\left(\xi_{1}^{p_{1}}\right)^{d_{3}}=1$ and also $\operatorname{gcd}\left(d_{1}, q_{1}\right)=1$ since $\operatorname{gcd}\left(p_{1}, q_{1}\right)=1$. Hence $\left(\xi_{N+1}^{d_{3}}\right)^{q_{1}}=$ 1 since $\xi_{1}^{p_{1}} \xi_{N+1}^{q_{1}}=1$. On the other hand $\left(\xi_{N+1}^{d_{3}}\right)^{d_{1}}=1$. Thus $\xi_{N+1}^{d_{3}}=1$ since $\operatorname{gcd}\left(d_{1}, q_{1}\right)=1$. Similarly we have $\xi_{N+1}^{d_{1}}=1$. Hence we have $\xi_{N+1}=1$ since $\operatorname{gcd}\left(d_{1}, d_{3}\right)=1$, and we have $\xi_{i}^{p_{i}}=1$ for all $i$ by Lemma 4.2 (1). By Lemma 3.4 (1), $\operatorname{gcd}\left(d_{1}, p_{i}\right)=1$ for $i \neq 1,2$, and $\operatorname{gcd}\left(d_{3}, p_{i}\right)=1$ for $i \neq 3,4$. Hence $\operatorname{gcd}\left(d, p_{i}\right)=1$ for $i \geq 5$. Therefore we have $\xi_{i}=1$ for $i \geq 5$, and $\xi_{1} \xi_{2} \xi_{3} \xi_{4}=1$. We note that $\operatorname{gcd}\left(d_{1}, p_{3}\right)=\operatorname{gcd}\left(d_{1}, p_{4}\right)=\operatorname{gcd}\left(d_{3}, p_{1}\right)=\operatorname{gcd}\left(d_{3}, p_{2}\right)=1$ are used in the next paragraph.

Since $\operatorname{gcd}\left(d_{3}, p_{1}\right)=1$ and $d_{1}$ divides $p_{1}$, we have $\operatorname{gcd}\left(d, p_{1}\right)=d_{1}$. Hence $\xi_{1}^{d}=1$ and $\xi_{1}^{p_{1}}=1$ imply $\xi_{1}^{d_{1}}=1$. Similarly we have $\xi_{2}^{d_{1}}=1, \xi_{3}^{d_{3}}=1$ and $\xi_{4}^{d_{3}}=1$. Thus $\xi_{1} \xi_{2}$ and $\xi_{3} \xi_{4}$ are $d_{1}$-th and $d_{3}$-th root of unities respectively. Hence we have $\xi_{1} \xi_{2}=\xi_{3} \xi_{4}=1$ since $\xi_{1} \xi_{2} \xi_{3} \xi_{4}=1$ and $\operatorname{gcd}\left(d_{1}, d_{3}\right)=1$. Hence $\psi_{d}\left(H_{1}(M)\right) \cong \mathbb{Z} / d \mathbb{Z}$ is generated by $\xi_{1}$ and $\xi_{3}$. For the similar reason as Step 1, both $\xi_{1}$ and $\xi_{3}$ are "primitive". Thus $\xi_{1}$ and $\xi_{2}$ (resp. $\xi_{3}$ and $\xi_{4}$ ) are primitive $d_{1}$-th (resp. $d_{3}-$ th) root of unities. Since $\operatorname{gcd}\left(p_{1}, q_{1}\right)=1$ and $d_{1}$ divides $p_{1}$, we have $\operatorname{gcd}\left(d_{1}, q_{1}\right)=1$. Similarly we have $\operatorname{gcd}\left(d_{1}, q_{2}\right)=\operatorname{gcd}\left(d_{3}, q_{3}\right)=\operatorname{gcd}\left(d_{3}, q_{4}\right)=1$. Hence we have

$$
\psi_{d}\left(\left[l_{i}^{\prime}\right]\right)=\xi_{i}^{-\bar{q}_{i}} \neq 1 \quad(i=1,2,3,4),
$$

where $\bar{q}_{1}, \bar{q}_{2}, \bar{q}_{3}$ and $\bar{q}_{4}$ are integers satisfying $q_{1} \bar{q}_{1} \equiv 1\left(\bmod d_{1}\right), q_{2} \bar{q}_{2} \equiv 1\left(\bmod d_{1}\right)$, $q_{3} \bar{q}_{3} \equiv 1\left(\bmod d_{3}\right)$ and $q_{4} \bar{q}_{4} \equiv 1\left(\bmod d_{3}\right)$ respectively. 
We set $M_{4}=E_{L} \cup V_{5} \cup \cdots \cup V_{N+1}$ and $\psi_{4, d}: \mathbb{Z}\left[H_{1}\left(M_{4}\right)\right] \rightarrow \mathbb{Q}\left(\zeta_{d}\right)$ as in Section 2 . By Lemma 4.3, we have

$$
\tau\left(M_{4}\right) \doteq\left(\left[m_{N+1}\right]-1\right)^{N-2} \prod_{i=5}^{N}\left(\left[l_{i}^{\prime}\right]-1\right)^{-1}=\left(\left[m_{N+1}\right]-1\right)^{2} \prod_{i=5}^{N}\left(\sum_{k=0}^{p_{i}-1}\left[l_{i}^{\prime}\right]^{k}\right),
$$

and hence $\tau^{\psi_{4, d}}\left(M_{4}\right)=0$. By Lemma 2.5 (3), we have

$$
\tau^{\psi_{d}}(M) \doteq \tau^{\psi_{4, d}}\left(M_{4}\right) \prod_{i=1}^{4}\left(\xi_{i}^{\bar{q}_{i}}-1\right)^{-1}=0
$$

and hence $\Delta_{K}\left(\zeta_{d}\right)=0$ by Lemma 2.6.

We next prove "only if" part of Theorem 1.3 (3). Suppose $\|M\|_{p}=0$. Then there exists a divisor $d \geq 2$ of $p$ such that $\Delta_{K}\left(\zeta_{d}\right)=0$, and there exists a pair $\{i, j\}$ such that $\operatorname{gcd}\left(p_{i}, p_{j}\right) \geq 2$ by (1). We may set $\{i, j\}=\{1,2\}$ without loss of generality. Let $\psi_{d}: \mathbb{Z}\left[H_{1}(M)\right] \rightarrow \mathbb{Q}\left(\zeta_{d}\right)$ be a ring homomorphism such that $\psi_{d}(T)=\zeta_{d}$, where $T$ is a generator of $H_{1}(M)$ that corresponds to a meridian of $K$. We set $\psi_{d}\left(\left[m_{i}\right]\right)=$ $\xi_{i}(i=1, \cdots, N+1)$, where $m_{i}$ is a meridian of $K_{i}$.

Step $3 \xi_{N+1}=1$.

Proof If $\psi_{d}\left(\left[l_{i}^{\prime}\right]\right)=1$ for some $i$, then we have $\xi_{N+1}=1$ by Lemma 4.2 (1) and (3), and $p_{i} s_{i}-q_{i} r_{i}=1$. Otherwise, since $\Delta_{K}\left(\zeta_{d}\right)=0$ and by Lemma 2.6, we have $\tau^{\psi_{d}}(M)=0$. Hence by Lemma 2.5 (1),

$$
\Delta_{L}\left(\xi_{1}, \cdots, \xi_{N+1}\right)=\left(\xi_{N+1}-1\right)^{N-1}=0
$$

Thus we have $\xi_{N+1}=1$.

Step 4 If $\operatorname{gcd}\left(d, p_{k}\right) \geq 2$, there is an index $h \neq k$ such that $\operatorname{gcd}\left(p_{k}, p_{h}\right) \geq 2$.

Proof Take a prime divisor $\ell$ of $\operatorname{gcd}\left(d, p_{k}\right)$. By Lemma 3.4 (2), there is an index $h \neq k$ such that $\ell$ is a divisor of $p_{h}$. Then $\ell$ is a divisor of $\operatorname{gcd}\left(p_{k}, p_{h}\right)$.

Step $5 d$ is not a common divisor of $p_{1}$ and $p_{2}$.

Proof If $d$ is a common divisor of $p_{1}$ and $p_{2}$, then $|M|_{d} \neq 0$ by Theorem 1.2 (2). This contradicts $\Delta_{K}\left(\zeta_{d}\right)=0$.

Step 6 There are indices $k \neq h$ such that $\{k, h\} \neq\{1,2\}$ and $\operatorname{gcd}\left(p_{k}, p_{h}\right) \geq 2$. 
Proof By Step 3 and Lemma 4.2 (1), we have $\xi_{k}^{p_{k}}=1$ for all $k$. Suppose $\operatorname{gcd}\left(d, p_{k}\right)=$ 1 for all $k \geq 3$. Then we have $\xi_{k}=1$ for all $k \geq 3$. By Lemma 4.2 (2), we have $\xi_{1} \xi_{2}=1$. Thus $\xi_{1}$ generates $\psi_{d}\left(H_{1}(M)\right) \cong \mathbb{Z} / d \mathbb{Z}$, and hence both $\xi_{1}$ and $\xi_{2}=\xi_{1}^{-1}$ are primitive $d$-th root of unities. Since $\xi_{1}^{p_{1}}=\xi_{2}^{p_{2}}=1, d$ is a common divisor of $p_{1}$ and $p_{2}$. This contradicts Step 5. Therefore there exists an index $k \geq 3$ such that $\operatorname{gcd}\left(d, p_{k}\right) \geq 2$. By Step 4, there exists an index $h \neq k \operatorname{such}$ that $\operatorname{gcd}\left(p_{k}, p_{h}\right) \geq 2$, and $\{k, h\} \neq\{1,2\}$ by $k \geq 3$.

This concludes the proof of Theorem 1.3.

\section{Proof of Theorem 1.4}

We set $f(t)=t^{2}-3 t+1$ in this section. Let $\alpha$ and $\beta(\alpha>\beta)$ be the roots of $f(t)=t^{2}-3 t+1=0$. Then we need the following lemmas on the norms of $f(t)$.

Lemma 5.1 $|f(t)|_{d}=\left|\Phi_{d}(\alpha) \Phi_{d}(\beta)\right|$, where

$$
\boldsymbol{\Phi}_{d}(x)=\prod_{i \in(\mathbb{Z} / d \mathbb{Z})^{\times}}\left(x-\zeta_{d}^{i}\right),
$$

the $d$-th cyclotomic polynomial.

Proof

$$
|f(t)|_{d}=\left|\prod_{i \in(\mathbb{Z} / d \mathbb{Z})^{\times}}\left(\zeta_{d}^{i}-\alpha\right)\left(\zeta_{d}^{i}-\beta\right)\right|=\left|\boldsymbol{\Phi}_{d}(\alpha) \boldsymbol{\Phi}_{d}(\beta)\right| .
$$

Lemma 5.2 Let $\ell$ be a prime number. Then we have the following:

(1) $|f(t)|_{\ell}=\alpha^{\ell}+\beta^{\ell}-2$.

(2) $|f(t)|_{\ell}>2$.

Proof (1) Since $\ell$ is prime, $\boldsymbol{\Phi}_{\ell}(x)=\left(x^{\ell}-1\right) /(x-1)$. Note that $\alpha+\beta=3$ and $\alpha \beta=1$. Hence by Lemma 5.1, we have

$$
|f(t)|_{\ell}=\left|\frac{\alpha^{\ell}-1}{\alpha-1} \cdot \frac{\beta^{\ell}-1}{\beta-1}\right|=\alpha^{\ell}+\beta^{\ell}-2 .
$$

(2) Since $\alpha=(3+\sqrt{5}) / 2=2.618 \cdots>2$, we have $|f(t)|_{\ell}=\alpha^{\ell}+\beta^{\ell}-2>2^{2}-2=$ 2 . 
Proof of Theorem 1.4 Let $\ell$ be a prime divisor of $p$. By Lemma 5.2, we have $|M|_{\ell}>2$. Hence $\|M\|_{p} \neq 1$ and $N \geq 3$ because $M$ is not a lens space. Since $f(t)=t^{2}-3 t+1$ is irreducible over $\mathbb{Q}$ and is not a cyclotomic polynomial, we have $f\left(\zeta_{d}\right) \neq 0$ for every positive integer $d$, and hence we have $\|M\|_{p} \neq 0$. By Theorem 1.3 , without loss of generality we may assume that

$$
\operatorname{gcd}\left(p_{1}, p_{2}\right) \geq 2 \text { and } \operatorname{gcd}\left(p_{i}, p_{j}\right)=1 \text { for }\{i, j\} \neq\{1,2\} .
$$

Step 1 Let $\ell$ be a prime divisor of $p$. Then $\ell$ is a divisor of $\operatorname{gcd}\left(p_{1}, p_{2}\right)$.

Proof Suppose $\ell$ divides none of $p_{i}$ 's. Then we have $|M|_{\ell}=|f(t)|_{\ell}=1$ by Theorem 1.1 (2). This contradicts Lemma 5.2 (2). Therefore $\ell$ is a divisor of some $p_{k}$. By Lemma 3.4 (2), there exists $h \neq k$ such that $\ell$ is a divisor of some $p_{h}$. By the assumption $(*)$, we have $\{k, h\}=\{1,2\}$, and hence $\ell$ is a divisor of $\operatorname{gcd}\left(p_{1}, p_{2}\right)$.

We first show that $\operatorname{gcd}\left(p_{1}, p_{2}\right)=2$ or 3 .

Step $2 \operatorname{gcd}\left(p_{1}, p_{2}\right)$ is not divisible by 4,6 nor 9 .

Proof Suppose that $\operatorname{gcd}\left(p_{1}, p_{2}\right)$ is divisible by 4 . Then $p$ is also divisible by 4 . By Theorem $1.2(2),|f(t)|_{2}=p_{3} \cdots p_{N}$ and $|f(t)|_{4}=\left(p_{3} \cdots p_{N}\right)^{2}$. By computing concretely, we have $|f(t)|_{2}=5$ and $|f(t)|_{4}=3^{2}$. This is a contradiction. Therefore $\operatorname{gcd}\left(p_{1}, p_{2}\right)$ is not divisible by 4 . Other cases are shown in a similar way.

Step 3 For any prime number $\ell \geq 5, \ell$ is not a divisor of $\operatorname{gcd}\left(p_{1}, p_{2}\right)$.

Proof Suppose $\ell$ is a divisor of $\operatorname{gcd}\left(p_{1}, p_{2}\right)$. Then $|M|_{\ell}=\left(p_{3} \cdots p_{N}\right)^{\ell-1}$ by Theorem 1.2 (2). Hence by Lemma 5.2 (2), there exists an integer $m \geq 2$ such that

$$
\alpha^{\ell}+\beta^{\ell}-2=m^{\ell-1}
$$

Since $\alpha^{x}+\alpha^{-x}-2^{x-1}-2>2^{x-1}-2, \alpha^{x}+\alpha^{-x}-2=2^{x-1}$ does not hold for $x \geq 5$. Hence we have $m \neq 2$.

By the equation (5-1), we have

$$
m^{\ell-1}<\alpha^{\ell} \text { and } m<\alpha^{\frac{\ell}{\ell-1}} .
$$

Suppose $\ell \geq 11$. Since

$$
\alpha^{11}<(2.62)^{11}=39931.4 \cdots<3^{10}=59049,
$$

we have $m<\alpha^{\frac{\ell}{\ell-1}} \leq \alpha^{\frac{11}{10}}<3$. By $m \neq 2$, this case does not occur. 
Suppose $\ell=5$ or 7 . Since

$$
\alpha^{5}<(2.62)^{5}=123.454 \cdots<4^{4}=256,
$$

we have $m<\alpha^{\frac{7}{6}}<\alpha^{\frac{5}{4}}<4$ and $m=3$.

If $\ell=5$, then we have $\alpha^{5}+\alpha^{-5}-2=121 \neq 3^{4}=81$.

If $\ell=7$, then $\alpha^{7}+\alpha^{-7}-2=841 \neq 3^{6}=729$, and we have Step 3 .

Step $4 \operatorname{gcd}\left(p_{1}, p_{2}\right)=2$ or 3 .

Proof By Step 2 and Step 3, we have the result.

We prove that $p=2$ or 3 .

Step $5 p$ is a power of 2 or 3 .

Proof By Step 1 and Step 4, we have the result.

Step $6 p$ is not divisible by 4 .

Proof Recall $|f(t)|_{4}=9$ (see Step 2). Suppose that $p$ is divisible by 4 . Then $|f(t)|_{4}=1$ as shown below, and this is a contradiction:

By Step 1 and Step 4 , we have $\operatorname{gcd}\left(p_{1}, p_{2}\right)=2$. Hence without loss of generality we may assume that $p_{1}$ is of the form $p_{1}=2 p_{1}^{\prime}$ where $p_{1}^{\prime}$ is odd. By Lemma 3.4 (1), $\operatorname{gcd}\left(p_{i}, 2\right)=1$ for $i \geq 3$, and hence we have the following claim:

Claim A $\operatorname{gcd}\left(p_{i}, 4\right)=1(3 \leq i \leq N)$.

We compute $\tau^{\psi_{4}}(M)$ by two ways. We use the same notation as in Section 4. By Lemma 2.6, we have

$$
\tau^{\psi_{4}}(M) \doteq f\left(\zeta_{4}\right)\left(\zeta_{4}-1\right)^{-1}\left(\zeta_{4}^{\bar{q}}-1\right)^{-1} .
$$

We note that at least one of $\xi_{1}, \cdots, \xi_{N+1}$ is a primitive 4-th root of unity because they generate $\psi_{4}\left(H_{1}(M)\right) \cong \mathbb{Z} / 4 \mathbb{Z}$. Suppose $\xi_{1}$ is not a primitive 4 -th root of unity. Then we have $\xi_{1}^{p_{1}}=\xi_{1}^{2} p_{1}^{\prime}=1$. Hence $\xi_{N+1}=1$ since $\operatorname{gcd}\left(4, q_{1}\right)=1$ and $\xi_{1}^{p_{1}} \xi_{N+1}^{q_{1}}=1$. Hence $\xi_{i}=1(3 \leq i \leq N)$ since $\xi_{i}^{p_{i}} \xi_{N+1}^{q_{i}}=1(3 \leq i \leq N), \xi_{N+1}=1$ and Claim A holds. By Lemma 4.2 (2), we have $\xi_{1} \xi_{2}=1$. Thus $\xi_{2}$ is also not a primitive $4-$ th root of unity. This is a contradiction. Therefore $\xi_{1}$ is a primitive 4-th root of unity. For the same reason, $\xi_{2}$ is also a primitive 4 -th root of unity. 
Since $\xi_{1}^{p_{1}} \xi_{N+1}^{q_{1}}=1\left(p_{1}=2 p_{1}^{\prime}, p_{1}^{\prime}:\right.$ odd, $q_{1}:$ odd $)$, we have $\xi_{N+1}=-1$. Since $\xi_{N+1}=-1$ and Claim A holds, we have

$$
\psi_{d}\left(\left[l_{i}^{\prime}\right]\right)=\xi_{i}^{r_{i}} \xi_{N+1}^{s_{i}}=\xi_{N+1}^{-\bar{p}_{i}}=-1(3 \leq i \leq N)
$$

(see the proof of Theorem $1.1(1)$ ). Since $r_{1}$ and $r_{2}$ are odd, both $\psi_{d}\left(\left[l_{1}^{\prime}\right]\right)$ and $\psi_{d}\left(\left[l_{2}^{\prime}\right]\right)$ are primitive $4-$ th root of unities. We set $\psi_{d}\left(\left[l_{i}^{\prime}\right]\right)=\zeta_{4}^{v_{i}}(i=1,2)$ where $\operatorname{gcd}\left(v_{i}, 4\right)=1$.

By Lemma 2.5 (1), we have

$$
\begin{aligned}
\tau^{\psi_{4}}(M) & \doteq(-1-1)^{N-1}\left(\zeta_{4}^{v_{1}}-1\right)^{-1}\left(\zeta_{4}^{v_{2}}-1\right)^{-1}(-1-1)^{-(N-1)} \\
& \doteq\left(\zeta_{4}^{v_{1}}-1\right)^{-1}\left(\zeta_{4}^{v_{1}}-1\right)^{-1}
\end{aligned}
$$

By (5-2) and (5-3), we have $|f(t)|_{4}=1$ as in the proof of Theorem 1.1 (2).

Step $7 \quad p$ is not divisible by 9 .

Proof By an easy computation, we have $|f(t)|_{9}=19^{2}$. Suppose that $p$ is divisible by 9. Then $|f(t)|_{9}=1$ as shown below, and this is a contradiction:

Suppose that $p$ is divisible by 9 . By Step 1 and Step 4 , we have $\operatorname{gcd}\left(p_{1}, p_{2}\right)=3$. Hence without loss of generality we may assume that $p_{1}$ is of the form $p_{1}=3 p_{1}^{\prime}$ where $\operatorname{gcd}\left(p_{1}^{\prime}, 3\right)=1$. By Lemma $3.4(1), \operatorname{gcd}\left(p_{i}, 3\right)=1$ for $i \geq 3$, and hence we have the following claim:

Claim B $\operatorname{gcd}\left(p_{i}, 9\right)=1(3 \leq i \leq N)$.

We compute $\tau^{\psi_{9}}(M)$ by two ways. By Lemma 2.6, we have

$$
\tau^{\psi_{9}}(M) \doteq f\left(\zeta_{9}\right)\left(\zeta_{9}-1\right)^{-1}\left(\zeta_{9}^{\bar{q}}-1\right)^{-1} .
$$

We note that at least one of $\xi_{1}, \cdots, \xi_{N+1}$ is a primitive 9-th root of unity because they generate $\psi_{9}\left(H_{1}(M)\right) \cong \mathbb{Z} / 9 \mathbb{Z}$. Suppose $\xi_{1}$ is not a primitive 9 -th root of unity. Then we have $\xi_{1}^{p_{1}}=\xi_{1}^{3 p_{1}^{\prime}}=1$. Hence $\xi_{N+1}=1$ since $\operatorname{gcd}\left(9, q_{1}\right)=1$ and $\xi_{1}^{p_{1}} \xi_{N+1}^{q_{1}}=1$. Hence $\xi_{i}=1(3 \leq i \leq N)$ since $\xi_{i}^{p_{i}} \xi_{N+1}^{q_{i}}=1(3 \leq i \leq N), \xi_{N+1}=1$ and Claim B holds. By Lemma 4.2 (2), we have $\xi_{1} \xi_{2}=1$. Thus $\xi_{2}$ is also not a primitive 9 -th root of unity. This is a contradiction. Therefore $\xi_{1}$ is a primitive $9-$ th root of unity. For the same reason, $\xi_{2}$ is also a primitive 9-th root of unity.

Since $\xi_{1}^{p_{1}} \xi_{N+1}^{q_{1}}=1$, we have that $\xi_{N+1}$ is a primitive 3-rd root of unity. Since $\xi_{N+1}$ is a primitive 3 -rd root of unity and Claim B holds, $\psi_{d}\left(\left[l_{i}^{\prime}\right]\right)=\xi_{i}^{r_{i}} \xi_{N+1}^{s_{i}}=\xi_{N+1}^{-\bar{p}_{i}}(3 \leq$ $i \leq N$ ) is a primitive 3-rd root of unity (see the proof of Theorem $1.1(1)$ ). Since $\operatorname{gcd}\left(r_{i}, 3\right)=1(i=1,2), \psi_{d}\left(\left[l_{i}^{\prime}\right]\right)(i=1,2)$ is a primitive 9 -th root of unity. We set 
$\psi_{d}\left(\left[l_{i}^{\prime}\right]\right)=\zeta_{9}^{v_{i}}(i=1,2), \psi_{d}\left(\left[l_{i}^{\prime}\right]\right)=\zeta_{3}^{w_{i}}(3 \leq i \leq N)$ and $\psi_{d}\left(\left[l_{N+1}^{\prime}\right]\right)=\zeta_{3}^{w}$ where $\operatorname{gcd}\left(v_{i}, 9\right)=1, \operatorname{gcd}\left(w_{i}, 3\right)=1$ and $\operatorname{gcd}(w, 3)=1$.

By Lemma 2.5 (1), we have

$$
\begin{aligned}
\tau^{\psi_{9}}(M) & \doteq\left(\zeta_{3}^{w}-1\right)^{N-1}\left(\zeta_{9}^{v_{1}}-1\right)^{-1}\left(\zeta_{9}^{v_{2}}-1\right)^{-1}\left(\zeta_{3}^{w}-1\right)^{-1} \prod_{i=3}^{N}\left(\zeta_{3}^{w_{i}}-1\right)^{-1} \\
& \doteq\left(\zeta_{3}^{w}-1\right)^{N-2}\left(\zeta_{9}^{v_{1}}-1\right)^{-1}\left(\zeta_{9}^{v_{2}}-1\right)^{-1} \prod_{i=3}^{N}\left(\zeta_{3}^{w_{i}}-1\right)^{-1}
\end{aligned}
$$

By (5-4) and (5-5), we have $|f(t)|_{9}=1$ as in the proof of Theorem 1.1 (2), which concludes the proof of Step 7.

We show finally the rest, concluding the proof of Theorem 1.4.

(i) By Theorem $1.2(3)$ and $|f(t)|_{2}=5$, we have $p_{3} \cdots p_{N}=5$. Hence $N=3$ and $p_{3}=5$.

(ii) By Theorem $1.2(3)$ and $|f(t)|_{3}=4^{2}$, we have $p_{3} \cdots p_{N}=4$. Then the case (a) $N=3$ and $p_{3}=4$ or the case (b) $N=4$ and $p_{3}=p_{4}=2$ occur. Since $\operatorname{gcd}\left(p_{i}, p_{j}\right)=1$ for $\{i, j\} \neq\{1,2\}$, the case (b) does not occur. Hence $N=3$ and $p_{3}=4$.

Acknowledgements The author would like to thank to Seiichi Kamada, Kimihiko Motegi, Makoto Sakuma, Koya Shimokawa, Masakazu Teragaito, Masaaki Ue and Yuichi Yamada for giving him useful advice. He would like to express special gratitude to Noriko Maruyama and Tsuyoshi Sakai for holding private seminars several times and reading carefully draft versions of the paper.

This paper is supported by the 21 st century COE program "Constitution of wide-angle mathematical basis focused on knots".

\section{References}

[1] S Boyer, D Lines, Surgery formulae for Casson's invariant and extensions to homology lens spaces, J. Reine Angew. Math. 405 (1990) 181-220 MR1041002

[2] M Brittenham, Y-Q Wu, The classification of exceptional Dehn surgeries on 2-bridge knots, Comm. Anal. Geom. 9 (2001) 97-113 MR1807953

[3] M Culler, C M Gordon, J Luecke, P B Shalen, Dehn surgery on knots, Ann. of Math. (2) 125 (1987) 237-300 MR881270

[4] G de Rham, Complexes à automorphismes et homéomorphie différentiable, Ann. Inst. Fourier Grenoble 2 (1950) 51-67 (1951) MR0043468 
[5] R Fintushel, R J Stern, Constructing lens spaces by surgery on knots, Math. Z. 175 (1980) 33-51 MR595630

[6] T Kadokami, Reidemeister torsion and lens surgeries on knots in homology spheres II, preprint

[7] T Kadokami, Reidemeister torsion and lens surgeries on knots in homology 3-spheres. I, Osaka J. Math. 43 (2006) 823-837 MR2303552

[8] T Kadokami, Y Yamada, Reidemeister torsion and lens surgeries on $(-2, m, n)$ pretzel knots, Kobe J. Math. 23 (2006) 65-78 MR2279859

[9] T Kadokami, Y Yamada, A deformation of the Alexander polynomials of knots yielding lens spaces, Bull. Austral. Math. Soc. 75 (2007) 75-89 MR2309549

[10] K Miyazaki, K Motegi, Seifert fibred manifolds and Dehn surgery, Topology 36 (1997) 579-603 MR1415607

[11] L Moser, Elementary surgery along a torus knot, Pacific J. Math. 38 (1971) 737-745 MR0383406

[12] A Némethi, L I Nicolaescu, Seiberg-Witten invariants and surface singularities, Geom. Topol. 6 (2002) 269-328 MR1914570

[13] L I Nicolaescu, The Reidemeister torsion of 3-manifolds, de Gruyter Studies in Mathematics 30, Walter de Gruyter \& Co., Berlin (2003) MR1968575

[14] P Orlik, Seifert manifolds, Lecture Notes in Mathematics 291, Springer, Berlin (1972) MR0426001

[15] T Sakai, Reidemeister torsion of a homology lens space, Kobe J. Math. 1 (1984) 47-50 MR784347

[16] N Saveliev, Invariants for homology 3-spheres, Encyclopaedia of Mathematical Sciences 140, Springer, Berlin (2002) MR1941324

[17] W P Thurston, The Geometry and Topology of Three-Manifolds, Princeton Univ. Math. Dept. Lecture Notes, Electronic Version 1.1 (2002) Available at www.msri.org/ publications/books/gt3m/

[18] V G Turaev, Reidemeister torsion in knot theory, Uspekhi Mat. Nauk 41 (1986) 97-147, 240 MR832411

[19] V Turaev, Introduction to combinatorial torsions, Lectures in Mathematics ETH Zürich, Birkhäuser Verlag, Basel (2001) MR1809561Notes taken by Felix Schlenk

Osaka City University Advanced Mathematical Institute

Sugimoto 3-3-138, Sumiyoshi-ku, Osaka 558-8585, Japan

kadokami@sci.osaka-cu.ac.jp

Received: 24 May 2007 Revised: 20 September 2007

Algebraic $6 \mathcal{G}$ Geometric $\mathcal{T}$ opology, Volume 7 (2007) 